\title{
Convergence of a space-time continuous Galerkin method for the wave equation
}

\section{Zhihui Zhao and Hong Li}

\section{"Correspondence:}

malhong@imu.edu.cn

School of Mathematical Sciences, Inner Mongolia University, No. 235,

West Road of University, Saihan

District, Hohhot, 010021, China

\section{Springer}

\begin{abstract}
This paper gives a new theoretical analysis of the space-time continuous Galerkin (STCG) method for the wave equation. We prove the existence and uniqueness of the numerical solutions and get optimal orders of convergence to numerical solutions regarding space that do not need any compatibility conditions on the space and time mesh size. Finally, we employ a numerical example to validate the effectiveness and feasibility of the STCG method.
\end{abstract}

MSC: 74S10; 65M15; 35Q35

Keywords: continuous Galerkin method; wave equation; optimal orders of convergence; numerical example

\section{Introduction}

In this article, we analyze the STCG method for the wave equation. As an example, we study the following model of the wave equation: find $u=u(x, t)$ satisfying

$$
\left\{\begin{array}{l}
u_{t t}-\Delta u=f(x, t), \quad(x, t) \in \Omega \times[0, T], \\
u(x, t)=0, \quad(x, t) \in \partial \Omega \times[0, T], \\
u(x, 0)=u_{0}(x), \quad u_{t}(x, 0)=v_{0}(x), \quad x \in \Omega,
\end{array}\right.
$$

where $\Omega$ is a bounded convex polygonal domain in $\mathbf{R}^{2}$ with boundary $\partial \Omega, u_{t t}=\partial^{2} u / \partial t^{2}$, $u_{t}=\partial u / \partial t$, and $T>0$ denotes the total time. The given body force $f$ and the initial value functions $u_{0}$ and $v_{0}$ are sufficiently smooth in order to ensure that the following theoretical analysis is effective.

The STCG method is a kind of finite element technique that uses continuous polynomial functions both in time and space to approximate the evolution problems, that is, it does not only use continuous polynomial functions to discretize space but also uses them to discretize time. Therefore, if we appropriately improve the degrees of polynomials with respect to time, we can easily derive any desired order of accuracy. Furthermore, in comparison with the theoretical analysis of the classical finite element methods in which the space is discretized by piecewise polynomial functions and the time is discretized by difference quotients, the theoretical analysis of such a method is uniform for any degree polynomials in time used to approximate the time variable. Especially, this method is more effective for solving wave problems since the corresponding discrete schemes retain the energy conservation properties (see [1-3]). Owing to the advantages mentioned, it has been applied

(c) Zhao and Li 2016. This article is distributed under the terms of the Creative Commons Attribution 4.0 International License (http://creativecommons.org/licenses/by/4.0/), which permits unrestricted use, distribution, and reproduction in any medium, provided you give appropriate credit to the original author(s) and the source, provide a link to the Creative Commons license, and indicate if changes were made. 
extensively to solve various kinds of time-related partial differential equations (TRPDEs) (see, e.g., [3-9]).

Aziz and Monk [4] applied the STCG method to investigate the approximation of the heat equation. French and Peterson [3, 8] and Bales and Lasiecka [9] also studied the approximation of the wave equation through the STCG method. However, the error estimates of [8] are obtained under the restrictive assumptions that the mesh size $h$ and time step $k$ must satisfy $k \leq \hat{c} h$, where $\hat{c}$ is a sufficiently small positive constant. For [3, 9], although the error analysis does not need any compatibility conditions between the space and time mesh size, the theoretical analysis is more abstract and complicated. The main purpose of this article is to propose a new kind of technique to give a theoretical analysis of the STCG method for the wave equation. To achieve this goal, we introduce Legendre polynomials and the corresponding Gauss integration rule and apply the basic properties of Lagrange interpolating polynomials fixed by the Legendre points to prove the existence and uniqueness of the numerical solutions; we also introduce a space-time projection operator to analyze the error estimates in $L^{2}$ and $H^{1}$ norms between the exact solutions and numerical solutions such that our theoretical analysis is more concise and understandable. Additionally, our error estimates also do not require the time step and space mesh size restriction. For [3, 9], the theoretical analysis is starting from an operator equation obtained by the coupled set of equations (2.10)-(2.11) by introducing an operator matrix. However, for most of the wave problems, there does not necessarily exist an operator matrix that can be used to make the coupled system become an operator equation. Thus, the theoretical analysis in $[3,9]$ have some limitations in some cases. Whereas the analysis showed in this article does not need introducing such an operator matrix, it is directly based on the coupled equation set (2.10)-(2.11) to study the existence, uniqueness, and convergence of the numerical solution. Therefore, compared with the methods presented in [3, 9], the idea employed in this paper is relatively easy to be applied to other wave problems. Based on our analysis, we think that the technique used is a kind of improvement and development for the existing papers (see $[3,8,9]$ ).

The rest of this paper is arranged in the following manners. In Section 2, we give some useful notation and definitions and propose the STCG method for the wave equation. In Section 3, we complete the error estimates in $L^{2}$ and $H^{1}$ norms for $u$ and $v$ without any restrictions on the space and time mesh size. In Section 4, a numerical example is given for illustrating the effectiveness and feasibility of the STCG method. Finally, in Section 5, we state the main conclusions and some perspectives.

\section{STCG method for 2D wave equation}

In this article, we use the standard definitions for Sobolev spaces and the corresponding norms (see [10]). For example, $\|\cdot\|_{s}$ and $|\cdot|_{s}$ denote the usual norms and seminorms of Sobolev spaces $H^{s}(\Omega)(s \geq 0)$. When $s=0$, the space $H^{0}(\Omega)$ is regarded as the space $L^{2}(\Omega)$, and by $(\cdot, \cdot)$ and $\|\cdot\|$ denote the corresponding inner product and norm. Furthermore, we define the energy norm on $L^{2}(\Omega) \times H^{1}(\Omega)$ by $\|(v, u)\|=\left\{\|v\|^{2}+\|\nabla u\|^{2}\right\}^{\frac{1}{2}}$. Let $H_{0}^{1}(\Omega)$ be the subspace of $H^{1}(\Omega)$ consisting of the functions in $H^{1}(\Omega)$ that vanish on $\partial \Omega$. In addition, the space $H^{l}\left(0, t_{n} ; H^{s}(\Omega)\right)$ and associated norms are defined by

$$
H^{l}\left(0, t_{n} ; H^{s}(\Omega)\right)=\left\{v(x, t) ; \sum_{j=0}^{l} \int_{0}^{t_{n}}\left\|\frac{\mathrm{d}^{j}}{\mathrm{~d} t} v(\cdot, t)\right\|_{s}^{2} \mathrm{~d} t<\infty\right\}
$$


and

$$
\|v\|_{H^{l}\left(0, t_{n} ; H^{s}\right)}=\left[\sum_{j=0}^{l} \int_{0}^{t_{n}}\left\|\frac{\mathrm{d}^{j}}{\mathrm{~d} t^{j}} v(\cdot, t)\right\|_{s}^{2} \mathrm{~d} t\right]^{1 / 2} .
$$

In particular, when $l=0$ and $s=0,1$, the associated norms are denoted by

$$
\|v\|_{L^{2}\left(0, t_{n} ; L^{2}\right)}=\left[\int_{0}^{t_{n}}\|v(\cdot, t)\|^{2} \mathrm{~d} t\right]^{1 / 2}
$$

and

$$
\|v\|_{L^{2}\left(0, t_{n} ; H^{1}\right)}=\left[\int_{0}^{t_{n}}\|v(\cdot, t)\|_{1}^{2} \mathrm{~d} t\right]^{1 / 2}
$$

where $t_{n}(n=1,2, \ldots, N)$ are the time nodes of the partition of time interval $[0, T]$, which will be defined later. If $t_{n}=T$, then $\|v\|_{H^{l}\left(0, t_{n} ; H^{s}\right)}$ are denoted by $\|v\|_{H^{l}\left(H^{s}\right)}$. Noting that $c$ in this paper is a general positive constant independent of all discretization parameters but may be different at different places.

Further, if we introduce the function $v=u_{t}$, then (1.1) can be rewritten as the first-order system concerning time

$$
\left\{\begin{array}{l}
v_{t}-\Delta u=f, \quad(x, t) \in \Omega \times[0, T], \\
v-u_{t}=0, \quad(x, t) \in \Omega \times[0, T], \\
u(x, t)=0, \quad(x, t) \in \partial \Omega \times[0, T], \\
u(x, 0)=u_{0}(x), \quad v(x, 0)=v_{0}(x), \quad x \in \Omega .
\end{array}\right.
$$

Let $U=H^{1}\left(0, T ; H_{0}^{1}(\Omega)\right)$. Then the weak formulation to (2.1) can be given as follows: find $(u, v) \in U \times U$ satisfying

$$
\begin{aligned}
& \int_{0}^{T}\left[\left(u_{t}, \phi_{t}\right)-\left(v, \phi_{t}\right)\right] \mathrm{d} t=0, \quad \forall \phi \in U, \\
& \int_{0}^{T}\left[\left(v_{t}, \psi_{t}\right)+\left(\nabla u, \nabla \psi_{t}\right)\right] \mathrm{d} t=\int_{0}^{T}\left(f, \psi_{t}\right) \mathrm{d} t, \quad \forall \psi \in U, \\
& u(x, 0)=u_{0}, \quad v(x, 0)=v_{0}, \quad x \in \Omega .
\end{aligned}
$$

In order to formulate the STCG method, let $\Im_{h}=\{K\}$ be a quasi-uniform triangulation partition of discrete region $\bar{\Omega}$ with $h=\max h_{K}$, where $h_{K}$ stands for the diameter of the triangle $K \in \Im_{h}$ (see [11-13]). Then, we introduce the subspace $S_{h m}(\Omega) \subset H_{0}^{1}(\Omega)$ consisting of piecewise continuous $m$ th-degree polynomials defined on the subdivision $\Im_{h}$ of $\Omega$ with mesh parameter $h$. Let $0=t_{0}<t_{1}<\cdots<t_{N}=T$ be a subdivision on time span $[0, T]$ with the maximum time step $k=\max _{1 \leq j \leq N}\left|t_{j}-t_{j-1}\right|$. Let $S_{k l}([0, T])$ be a finite element space on this subdivision consisting of continuous piecewise $l$ th-degree polynomials regarding time, i.e., $S_{k l}([0, T])=\left\{v \in C^{0}([0, T]):\left.v\right|_{\left[t_{j-1}, t_{j}\right]} \in P_{l}\left(\left[t_{j-1}, t_{j}\right]\right), j=1, \ldots, N\right\}$, where $P_{l}\left(\left[t_{j-1}, t_{j}\right]\right)$ is the set of polynomials of degree $l$ restricted on the interval $\left[t_{j-1}, t_{j}\right]$. Finally, we define $U_{h k}=S_{h m}(\Omega) \otimes S_{k l}([0, T])$. Then, the STCG formulation to the wave equation is given as 
follows: find $\left(u^{h k}, v^{h k}\right) \in U_{h k}^{2}$ such that

$$
\begin{aligned}
& \int_{0}^{T}\left[\left(u_{t}^{h k}, \phi_{t}\right)-\left(v^{h k}, \phi_{t}\right)\right] \mathrm{d} t=0, \quad \forall \phi \in U_{h k}, \\
& \int_{0}^{T}\left[\left(v_{t}^{h k}, \psi_{t}\right)+\left(\nabla u^{h k}, \nabla \psi_{t}\right)\right] \mathrm{d} t=\int_{0}^{T}\left(f, \psi_{t}\right) \mathrm{d} t, \quad \forall \psi \in U_{h k}, \\
& u^{h k}(x, 0)=P_{h} u_{0}, \quad v^{h k}(x, 0)=P_{h} v_{0}, \quad x \in \Omega .
\end{aligned}
$$

The STCG solution pair $\left(u^{h k}, v^{h k}\right)$ can be computed by marching through successive time levels. To see this, let $J_{n}=\left[t_{n}, t_{n+1}\right]$, and let $P_{l}\left(J_{n}\right)$ denote the set of polynomials of degree $l$ defined on interval $J_{n}$. Then, for $n=0,1, \ldots, N-1$, we find the STCG solution pair $\left(u^{h k}, v^{h k}\right)$ on $J_{n}$ satisfying

$$
\begin{aligned}
& \int_{J_{n}}\left[\left(u_{t}^{h k}, \phi_{t}\right)-\left(v^{h k}, \phi_{t}\right)\right] \mathrm{d} t=0, \quad \forall \phi \in S_{h m}(\Omega) \otimes P_{l}\left(J_{n}\right), \\
& \int_{J_{n}}\left[\left(v_{t}^{h k}, \psi_{t}\right)+\left(\nabla u^{h k}, \nabla \psi_{t}\right)\right] \mathrm{d} t=\int_{J_{n}}\left(f, \psi_{t}\right) \mathrm{d} t, \quad \forall \psi \in S_{h m}(\Omega) \otimes P_{l}\left(J_{n}\right)
\end{aligned}
$$

or, equivalently,

$$
\begin{aligned}
& \int_{J_{n}}\left[\left(u_{t}^{h k}, \phi\right)-\left(v^{h k}, \phi\right)\right] \mathrm{d} t=0, \quad \forall \phi \in S_{h m}(\Omega) \otimes P_{l-1}\left(J_{n}\right), \\
& \int_{J_{n}}\left[\left(v_{t}^{h k}, \psi\right)+\left(\nabla u^{h k}, \nabla \psi\right)\right] \mathrm{d} t \\
& =\int_{J_{n}}(f, \psi) \mathrm{d} t, \quad \forall \psi \in S_{h m}(\Omega) \otimes P_{l-1}\left(J_{n}\right),
\end{aligned}
$$

with $u^{h k}(x, 0)=P_{h} u_{0}, v^{h k}(x, 0)=P_{h} v_{0}$ (where $P_{h}$ is the elliptic projection defined further in (3.1)), and $u^{h k}\left(x, t_{n}\right), v^{h k}\left(x, t_{n}\right)(n=0,1, \ldots, N-1, x \in \Omega)$ have been fixed at previous time level.

Remark 1 In fact, (2.8)-(2.9) can be regarded as the Petrov-Galerkin method since they are tested by $\phi_{t}$ and $\psi_{t}$, respectively.

To analyze the well-posedness of problem (2.10)-(2.11), we need to introduce the Legendre polynomials and the corresponding Gauss integration rule. For a given $l \geq 1$, let $\left\{\ell_{i}\right\}_{i=1}^{l}$ be the Lagrange interpolating polynomials of degree $l-1$, that is,

$$
\ell_{i}(\mu)=\prod_{j=1, j \neq i}^{l} \frac{\left(\mu-\mu_{j}\right)}{\left(\mu_{i}-\mu_{j}\right)},
$$

where the interpolating points $0<\mu_{1}<\cdots<\mu_{l}<1$ are $l$ roots of the Legendre polynomial on the interval $[0,1]$. Then the Gauss-Legendre integration formula is as follows:

$$
\int_{0}^{1} m(\mu) \mathrm{d} \mu \cong \sum_{j=1}^{l} \omega_{j} m\left(\mu_{j}\right),
$$

where $\omega_{j}=\int_{0}^{1} \ell_{j}(\mu) \mathrm{d} \mu$, which is exact for any polynomial of degree not higher than $2 l-1$. 
Further, applying the linear transformation $t=t_{n}+\mu k_{n}\left(k_{n}=t_{n+1}-t_{n}\right)$ that maps the unit interval $[0,1]$ onto the interval $J_{n}$, its quadrature points and weights are defined by

$$
\left\{\begin{array}{l}
t_{n, i}=t_{n}+\mu_{i} k_{n}, \quad i=1, \ldots, l \\
\ell_{n, i}(t)=\ell_{i}(\mu) \\
\omega_{n, i}=\int_{t_{n}}^{t_{n+1}} \ell_{n, i}(t) \mathrm{d} t=k_{n} \int_{0}^{1} \ell_{i}(\mu) \mathrm{d} \mu=k_{n} \omega_{i}, \quad i=1, \ldots, l .
\end{array}\right.
$$

Then the Gauss-Legendre integration formula associated with $J_{n}$ is as follows:

$$
\int_{t_{n}}^{t_{n+1}} m(t) \mathrm{d} t \cong \sum_{i=1}^{l} \omega_{n, i} m\left(t_{n, i}\right)
$$

We also need to employ the Lagrange interpolating polynomials $\left\{\tilde{\ell}_{i}\right\}_{i=0}^{l}$ of degree $l$ corresponding to the $l+1$ interpolating points $0=\mu_{0}<\mu_{1}<\cdots<\mu_{l}<1$, that is,

$$
\tilde{\ell}_{i}(\mu)=\prod_{j=0, j \neq i}^{l} \frac{\left(\mu-\mu_{j}\right)}{\left(\mu_{i}-\mu_{j}\right)}
$$

Choosing $\left\{\tilde{\ell}_{n, i}\right\}_{i=0}^{l}$ (where $\left.\tilde{\ell}_{n, i}(t)=\tilde{\ell}_{i}(\mu)\right)$ as the basis functions to the polynomial space $P_{l}\left(J_{n}\right)$, then $\left.\left(u^{h k}, v^{h k}\right)\right|_{J_{n}}$ are solely fixed by the functions $\left(u_{n, i}^{h k}, v_{n, i}^{h k}\right)=\left(u^{h k}\left(x, t_{n . i}\right), v^{h k}\left(x, t_{n . i}\right)\right) \in$ $S_{h m} \times S_{h m}$ such that

$$
u^{h k}(x, t)=\sum_{i=0}^{l} \tilde{\ell}_{n, i}(t) u_{n, i}^{h k}(x), \quad v^{h k}(x, t)=\sum_{i=0}^{l} \tilde{\ell}_{n, i}(t) v_{n, i}^{h k}(x), \quad(x, t) \in \Omega \times J_{n},
$$

where $t_{n, 0}=t_{n}$.

Further, let $\left(u_{n, i}^{h k}, v_{n, i}^{h k}\right)=\left(\tilde{u}_{n, i}^{h k} \mu_{i}^{1 / 2}, \tilde{v}_{n, i}^{h k} \mu_{i}^{1 / 2}\right)(i=1, \ldots, l)$. Then the new expressions for $u^{h k}(x, t)$ and $v^{h k}(x, t)$ can be rewritten as

$$
\begin{aligned}
u^{h k}(x, t) & =\sum_{i=0}^{l} \tilde{\ell}_{n, i}(t) u_{n, i}^{h k}(x) \\
& =\sum_{i=1}^{l} \mu_{i}^{1 / 2} \tilde{\ell}_{n, i}(t) \tilde{u}_{n, i}^{h k}(x)+\tilde{\ell}_{n, 0}(t) u_{n, 0}^{h k}(x), \\
v^{h k}(x, t) & =\sum_{i=0}^{l} \tilde{\ell}_{n, i}(t) v_{n, i}^{h k}(x) \\
& =\sum_{i=1}^{l} \mu_{i}^{1 / 2} \tilde{\ell}_{n, i}(t) \tilde{v}_{n, i}^{h k}(x)+\tilde{\ell}_{n, 0}(t) v_{n, 0}^{h k}(x) .
\end{aligned}
$$

By choosing $(\phi, \psi)=\left(\mu_{i}^{-1 / 2} \ell_{n, i} \varphi_{1}, \mu_{i}^{-1 / 2} \ell_{n, i} \varphi_{2}\right)$ (where $\left.\varphi_{i} \in S_{h m}, i=1,2\right)$ in (2.10) and (2.11) we can equivalently rewrite the STCG scheme (2.10)-(2.11) for $\left(\tilde{u}_{n, j}^{h k} \tilde{v}_{h, j}^{h k}\right)(j=1,2, \ldots, l)$ as

$$
\left\{\begin{array}{l}
\sum_{j=1}^{l} \tilde{b}_{i j}\left(\tilde{u}_{n, j}^{h k}, \varphi_{1}\right)-k_{n} \omega_{i}\left(\tilde{v}_{n, i}^{h k}, \varphi_{1}\right)=-\mu_{i}^{-1 / 2} b_{i 0}\left(u_{n, 0}^{h k}, \varphi_{1}\right), \\
\sum_{j=1}^{l} \tilde{b}_{i j}\left(\tilde{v}_{n, j}^{k}, \varphi_{2}\right)+k_{n} \omega_{i}\left(\nabla \tilde{u}_{n, i}^{h k}, \nabla \varphi_{2}\right) \\
\quad=-\mu_{i}^{-1 / 2} b_{i 0}\left(v_{n, 0}^{h k}, \varphi_{2}\right)+\int_{J_{n}} \mu_{i}^{-1 / 2} \ell_{n, i}\left(f, \varphi_{2}\right) \mathrm{d} t, \quad i=1, \ldots, l,
\end{array}\right.
$$


where

$$
\tilde{b}_{i j}=\mu_{i}^{-1 / 2} b_{i j} \mu_{j}^{1 / 2}, \quad b_{i j}=\int_{J_{n}} \tilde{\ell}_{n, j}^{\prime}(t) \ell_{n, i}(t) \mathrm{d} t, \quad i=1, \ldots, l, j=0,1, \ldots, l .
$$

Remark 2 To illustrate how to use (2.18) to solve the numerical solution, we take $l=2$ for example. In this case, $\tilde{\ell}_{n, j}(t)(j=0,1,2)$ is determined via $t_{n, 0}, t_{n, 1}$, and $t_{n, 2}$ or via (2.14), which is equivalently fixed by $0, \mu_{1}$, and $\mu_{2}$, and $\ell_{n, i}(i=1,2)$ is fixed via $t_{n, 1}, t_{n, 2}\left(\right.$ or $\left.\mu_{1}, \mu_{2}\right)$, where $\mu_{1}, \mu_{2}$ are the two roots of the quadratic Lengendre polynomial defined in interval $[0,1]$. Therefore, by $(2.19)$ we can obtain the values of $\tilde{b}_{i j}(i, j=1,2)$ and $b_{i 0}(i=1,2)$. In addition, since $\tilde{u}_{n, j}^{h k}$ and $\tilde{v}_{n, j}^{h k}$ are elements of $S_{h m}$, they can be expressed as follows:

$$
\begin{aligned}
& \tilde{u}_{n, j}^{h k}=c_{j, 1} \chi_{1}+c_{j, 2} \chi_{2}+\cdots+c_{j, n} \chi_{n}, \\
& \tilde{v}_{n, j}^{h k}=d_{j, 1} \chi_{1}+d_{j, 2} \chi_{2}+\cdots+d_{j, n} \chi_{n},
\end{aligned}
$$

where $\chi_{i}(i=1,2, \ldots, n)$ denote the basis functions of $S_{h m}$, and $n$ stands for the dimension of $S_{h m}$. Then we substitute (2.20) into (2.18) and let $\varphi_{1}$ and $\varphi_{2}$ take $\chi_{i}(i=1,2, \ldots, n)$, respectively. We finally obtain the set of equations

$$
\left(\begin{array}{cccc}
k_{n} \omega_{1} A & 0 & -\tilde{b}_{11} A & -\tilde{b}_{12} A \\
0 & k_{n} \omega_{2} A & -\tilde{b}_{21} A & -\tilde{b}_{22} A \\
\tilde{b}_{11} A & \tilde{b}_{12} A & k_{n} \omega_{1} M & 0 \\
\tilde{b}_{21} A & \tilde{b}_{22} A & 0 & k_{n} \omega_{2} M
\end{array}\right)\left(\begin{array}{l}
d_{1} \\
d_{2} \\
c_{1} \\
c_{2}
\end{array}\right)=\left(\begin{array}{l}
f_{1} \\
f_{2} \\
g_{1} \\
g_{2}
\end{array}\right),
$$

where $A=\left(a_{i j}\right)_{n \times n}, a_{i j}=\left(\chi_{i}, \chi_{j}\right), M=\left(m_{i j}\right)_{n \times n}, m_{i j}=\left(\nabla \chi_{i}, \nabla \chi_{j}\right) . c_{i}=\left(c_{i, 1}, c_{i, 2}, \ldots, c_{i, n}\right)^{T}$, $d_{i}=\left(d_{i, 1}, d_{i, 2}, \ldots, d_{i, n}\right)^{T}(i=1,2) \cdot f_{i}=\left(f_{i, 1}, f_{i, 2}, \ldots, f_{i, n}\right)^{T}, g_{i}=\left(g_{i, 1}, g_{i, 2}, \ldots, g_{i, n}\right)^{T}(i=1,2), f_{i, j}=$ $-\mu_{i}^{-1 / 2} b_{i 0}\left(u_{n, 0}^{h k}, \chi_{j}\right), g_{i, j}=-\mu_{i}^{-1 / 2} b_{i 0}\left(v_{n, 0}^{h k}, \chi_{j}\right)+\int_{J_{n}} \mu_{i}^{-1 / 2} \ell_{n, i}\left(f, \chi_{j}\right) \mathrm{d} t(j=1,2, \ldots, n)$. We notice that $u_{n, 0}^{h k}$ and $v_{n, 0}^{h k}$ have been found in the previous time level. Therefore, by (2.21) and (2.16), (2.17) we can obtain $u^{h k}(x, t)$ and $v^{h k}(x, t)$.

Furthermore, the following lemma holds (see [14]).

Lemma 1 Let $\lambda:=\frac{1}{2} \min _{j} \frac{\omega_{j}}{\mu_{j}}$. Then

$$
x^{T} \widetilde{\mathscr{B}} x \geq \lambda|x|^{2}=\lambda\left(\sum_{i=1}^{l} x_{i}^{2}\right), \quad \forall x \in R^{l},
$$

where $\widetilde{\mathscr{B}}=D^{-1 / 2} \mathscr{B} D^{1 / 2}, \mathscr{B}=\left(b_{i j}\right)_{l \times l}$, and $D=\operatorname{diag}\left\{\mu_{1}, \ldots, \mu_{l}\right\}$.

For further theoretical analysis, we need to introduce the discrete operator $A_{h}: L^{2}(\Omega) \rightarrow$ $S_{\text {hm }}(\Omega)$ defined by

$$
\left(\nabla A_{h} u, \nabla \phi\right)=(u, \phi), \quad \forall \phi \in S_{h m}(\Omega) .
$$

From this definition we can easily prove that $A_{h}$ is a nonnegative self-adjoint operator. Therefore, there exists a square-root operator $A_{h}^{1 / 2}$ satisfying $\left(u, A_{h} u\right)=\left(A_{h}^{1 / 2} u, A_{h}^{1 / 2} u\right)(\forall u \in$ $\left.L^{2}(\Omega)\right)$. Furthermore, $A_{h}$ is invertible on $S_{h m}$. In fact, if we take $A_{h} \phi=0$ for arbitrary $\phi \in$ 
$S_{h m}$, then $(\phi, \phi)=\left(\nabla A_{h} \phi, \nabla \phi\right)=0$, that is, $\phi=0$. Note that $A_{h}$ can also be extended to functions in $L^{2}\left(0, T ; L^{2}(\Omega)\right)$ in the $L^{2}$ sense.

Theorem 1 Assume that the solution pair $\left(u^{h k}\left(t_{n}\right), v^{h k}\left(t_{n}\right)\right)$ is given in the time level $J_{n-1}$. Then, for $k$ small enough, there exists a unique solution pair $\left(u^{h k}, v^{h k}\right) \in\left(S_{h m}(\Omega) \otimes P_{l}\left(J_{n}\right)\right)^{2}$ to the system of equation (2.10)-(2.11).

Proof Since (2.10)-(2.11) is a linear system of equations, in order to demonstrate the existence and uniqueness of its approximate solutions, we only need to prove that if $f=0$ and $u_{n, 0}^{h k}=v_{n, 0}^{h k}=0$, then there is a zero solution pair to it.

Taking $\left(\varphi_{1}, \varphi_{2}\right)=\left(\tilde{u}_{n, i}^{h k}, A_{h} \tilde{v}_{n, i}^{h k}\right)$ in (2.18), we have

$$
\begin{aligned}
\sum_{i, j=1}^{l} \tilde{b}_{i j}\left\{\left(\tilde{u}_{n, j}^{h k}, \tilde{u}_{n, i}^{h k}\right)+\left(A_{h}^{1 / 2} \tilde{v}_{n, j}^{h k}, A_{h}^{1 / 2} \tilde{v}_{n, i}^{h k}\right)\right\} \\
=-\sum_{i=1}^{l} \mu_{i}^{-1 / 2} b_{i 0}\left\{\left(u_{n, 0}^{h k}, \tilde{u}_{n, i}^{h k}\right)+\left(A_{h}^{1 / 2} v_{n, 0}^{h k}, A_{h}^{1 / 2} \tilde{v}_{n, i}^{h k}\right)\right\} \\
\quad+\sum_{i=1}^{l} \int_{J_{n}} \mu_{i}^{-1 / 2} \ell_{n, i}\left(A_{h}^{1 / 2} f, A_{h}^{1 / 2} \tilde{v}_{n, i}^{h k}\right) \mathrm{d} t .
\end{aligned}
$$

For the terms of the left side of (2.24), in view of Lemma 1, we have

$$
\lambda \sum_{i=1}^{l}\left(\left\|\tilde{u}_{n, i}^{h k}\right\|^{2}+\left\|A_{h}^{1 / 2} \tilde{v}_{n, i}^{h k}\right\|^{2}\right) \leq \sum_{i, j=1}^{l} \tilde{b}_{i j}\left\{\left(\tilde{u}_{n, j}^{h k} \tilde{u}_{n, i}^{h k}\right)+\left(A_{h}^{1 / 2} \tilde{v}_{n, j}^{h k}, A_{h}^{1 / 2} \tilde{v}_{n, i}^{h k}\right)\right\} .
$$

For the first part of the right side of (2.24), using the Hölder and Cauchy inequalities, we have

$$
\begin{aligned}
& \sum_{i=1}^{l} \mu_{i}^{-1 / 2} b_{i 0}\left\{\left(u_{n, 0}^{h k}, \tilde{u}_{n, i}^{h k}\right)+\left(A_{h}^{1 / 2} v_{n, 0}^{h k}, A_{h}^{1 / 2} \tilde{v}_{n, i}^{h k}\right)\right\} \\
& \quad \leq \sum_{i=1}^{l} \mu_{i}^{-1 / 2} \int_{0}^{1} \tilde{\ell}_{0}^{\prime}(\mu) \ell_{i}(\mu) \mathrm{d} \mu\left(\left\|u_{n, 0}^{h k}\right\|\left\|\tilde{u}_{n, i}^{h k}\right\|+\left\|A_{h}^{1 / 2} v_{n, 0}^{h k}\right\|\left\|A_{h}^{1 / 2} \tilde{u}_{n, i}^{h k}\right\|\right) \\
& \quad \leq \frac{c}{2 \lambda} \sum_{i=1}^{l}\left(\left\|u_{n, 0}^{h k}\right\|^{2}+\left\|A_{h}^{1 / 2} v_{n, 0}^{h k}\right\|^{2}\right)+\frac{\lambda}{2} \sum_{i=1}^{l}\left(\left\|\tilde{u}_{n, i}^{h k}\right\|^{2}+\left\|A_{h}^{1 / 2} \tilde{v}_{n, i}^{h k}\right\|^{2}\right) .
\end{aligned}
$$

Also, by the Hölder and Cauchy inequalities, noting that $\int_{J_{n}} \ell_{n, i}^{2}(t) \mathrm{d} t=k_{n} \omega_{i}$, for the second part of the right side of (2.24), we derive

$$
\begin{aligned}
& \sum_{i=1}^{l} \int_{J_{h}} \mu_{i}^{-1 / 2} \ell_{n, i}\left(A_{h}^{1 / 2} f, A_{h}^{1 / 2} \tilde{v}_{n, i}^{h k}\right) \mathrm{d} t \\
& \quad \leq c\left\|A_{h}^{1 / 2} f\right\|_{L^{2}\left(J_{n} ; L^{2}\right)}^{2}+c k \sum_{i=1}^{l}\left\|A_{h}^{1 / 2} \tilde{v}_{n, i}^{h k}\right\|^{2} .
\end{aligned}
$$


Combining (2.24) with (2.25)-(2.27) and assuming that $k \leq \frac{\lambda}{4 c}$, we have

$$
\begin{aligned}
& \frac{\lambda}{4} \sum_{i=1}^{l}\left(\left\|\tilde{u}_{n, i}^{h k}\right\|^{2}+\left\|A_{h}^{1 / 2} \tilde{v}_{n, i}^{h k}\right\|^{2}\right) \\
& \quad \leq \frac{\lambda}{2} \sum_{i=1}^{l}\left\|\tilde{u}_{n, i}^{h k}\right\|^{2}+\left(\frac{\lambda}{2}-c k\right) \sum_{i=1}^{l}\left\|A_{h}^{1 / 2} \tilde{v}_{n, i}^{h k}\right\|^{2} \\
& \quad \leq \frac{c}{2 \lambda} \sum_{i=1}^{l}\left(\left\|u_{n, 0}^{h k}\right\|^{2}+\left\|A_{h}^{1 / 2} v_{n, 0}^{h k}\right\|^{2}\right)+c\left\|A_{h}^{1 / 2} f\right\|_{L^{2}\left(J_{n} ; L^{2}\right)}^{2}
\end{aligned}
$$

Setting $f=0$ and $u_{n, 0}^{h k}=v_{n, 0}^{h k}=0$ in (2.28), since $A_{h}^{1 / 2}$ invertible on $S_{h m}$, we get $\tilde{u}_{n, i}^{h k}=\tilde{v}_{n, i}^{h k}=0$ $(i=1,2, \ldots, l)$, that is, $u^{h k}=v^{h k}=0$. Because the existence of the solution is implied by the uniqueness, system (2.10)-(2.11) has a unique solution pair.

\section{Error estimates of the STCG solutions}

In this section, we give some error estimates between the approximate solutions and exact solutions. To this end, we need to introduce some projections.

We define the space variable Ritz projection $P_{h}: H_{0}^{1}(\Omega) \rightarrow S_{h m}(\Omega)$, that is, for $u \in H_{0}^{1}(\Omega)$, we have

$$
\left(\nabla P_{h} u, \nabla \phi_{h}\right)=\left(\nabla u, \nabla \phi_{h}\right), \quad \forall \phi_{h} \in S_{h m}(\Omega)
$$

Because of the regularity of the triangulation $\Im_{h}$, we know that $P_{h}$ has the following approximation properties (see $[11,12]$ ). If $u \in H_{0}^{1}(\Omega) \cap H^{s}(\Omega)$, then

$$
\left\|P_{h} u-u\right\|_{r} \leq c h^{s-r}\|u\|_{s}, \quad 1 \leq s \leq m+1 ; r=0,1
$$

The projection $P_{h}$ can be generalized to functions of $t$ and $x$ in the $L^{2}$ sense. Namely, we define the generalized projection $P_{h}: L^{2}\left(0, T ; H_{0}^{1}(\Omega)\right) \rightarrow S_{h m}(\Omega) \times L^{2}(0, T)$ by

$$
\int_{0}^{T}\left(\nabla P_{h} u, \nabla \phi_{h}\right) \mathrm{d} t=\int_{0}^{T}\left(\nabla u, \nabla \phi_{h}\right) \mathrm{d} t, \quad \forall \phi \in S_{h m}(\Omega) \otimes L^{2}(0, T) .
$$

Furthermore, we need to define the time projection $P_{k}: H^{1}(0, T) \rightarrow S_{k l}([0, T])$ as well; namely, for $w \in H^{1}(0, T)$, it satisfies $P_{k} w(0)=w(0)$ and

$$
\int_{0}^{T}\left(P_{k} w\right)_{t} \phi_{t}^{k} \mathrm{~d} t=\int_{0}^{T} w_{t} \phi_{t}^{k} \mathrm{~d} t, \quad \forall \phi^{k} \in S_{k l}([0, T]) .
$$

We can easily conclude from the classical FE techniques that $P_{k}$ satisfies the following estimate: for $w \in H^{1}(0, T) \cap H^{s}(0, T)$,

$$
\left\|P_{k} w-w\right\|_{H^{r}(0, T)} \leq c h^{s-r}\|w\|_{H^{s}(0, T)}, \quad-l+1 \leq r \leq 1 \leq s \leq l+1 .
$$

Also, we can generalize $P_{k}$ to functions of $t$ and $x$ in the $L^{2}$ sense. Thus, we define $P_{k}$ : $H^{1}\left(0, T ; L^{2}(\Omega)\right) \rightarrow L^{2}(\Omega) \times S_{k l}([0, T])$ by

$$
\int_{0}^{T}\left(\left(P_{k} w\right)_{t}, \phi_{t}^{k}\right) \mathrm{d} t=\int_{0}^{T}\left(w_{t}, \phi_{t}^{k}\right) \mathrm{d} t, \quad \forall \phi^{k} \in L^{2}(\Omega) \otimes S_{k l}([0, T])
$$


with initial condition $\left(P_{k} w(0), \phi\right)=(w(0), \phi)\left(\forall \phi \in L^{2}(\Omega)\right)$. Further, from (3.6) we conclude that $P_{k} w\left(t_{n}\right)=w\left(t_{n}\right)(n=0,1,2, \ldots, N)$. Moreover, the projections $P_{h}$ and $P_{k}$ satisfy the following properties (see [4]).

Lemma 2 Let $P_{h}$ and $P_{k}$ be defined in the generalized sense via (3.3) and (3.6).

(1) Let $v \in H^{2}\left(0, T ; H^{2}(\Omega)\right)$. Then

$$
\begin{aligned}
& \left(P_{h} v\right)_{t}=P_{h} v_{t}, \quad \nabla\left(P_{k} v\right)=P_{k} \nabla v, \\
& P_{h} P_{k} v=P_{k} P_{h} v, \quad A_{h} P_{k} v=P_{k} A_{h} v .
\end{aligned}
$$

(2) Let $v \in H^{s}\left(0, t_{n} ; L^{2}(\Omega)\right)$. Then, for $-l+1 \leq r \leq 1 \leq s \leq l+1$,

$$
\int_{\Omega} \sum_{m=0}^{n-1}\left\|v-P_{k} v\right\|_{H^{r}\left(J_{m}\right)}^{2} \mathrm{~d} x \leq c k^{2(s-r)}\|v\|_{H^{s}\left(0, t_{n} ; L^{2}\right)^{2}}^{2} .
$$

(3) Let $v \in H^{1}\left(0, T ; H^{m+1}(\Omega)\right) \cap H^{1}\left(0, T ; H_{0}^{1}(\Omega)\right)$ for all $t \in[0, T]$. Then

$$
\left\|\left(v-P_{h} v\right)(t)\right\|_{r} \leq c h^{m+1-r}\|v(t)\|_{m+1}, \quad r=0,1 .
$$

(4) Let $v \in L^{2}\left(0, t_{n} ; H^{s}(\Omega)\right) \cap H^{1}\left(0, t_{n} ; H_{0}^{1}(\Omega)\right)$. Then

$$
\left\|\left(v-P_{h} v\right)(t)\right\|_{L^{2}\left(0, t_{n} ; L^{2}\right)} \leq c h^{s}\|v(t)\|_{L^{2}\left(0, t_{n} ; H^{s}\right)}, \quad 1 \leq s \leq m+1 .
$$

(5) Let $v \in H^{l+1}\left(0, t_{n} ; L^{2}(\Omega)\right) \cap H^{1}\left(0, t_{n} ; H_{0}^{1}(\Omega)\right)$ and $v_{t} \in L^{2}\left(0, t_{n} ; H^{m+1}(\Omega)\right) \cap H^{1}\left(0, t_{n} ; H_{0}^{1}(\Omega)\right)$. Then

$$
\left\|\left(v-P_{h} P_{k} v\right)_{t}\right\|_{L^{2}\left(0, t_{n} ; L^{2}\right)} \leq c\left\{h^{m+1}\left\|v_{t}\right\|_{L^{2}\left(0, t_{n} ; H^{m+1}\right)}+k^{l}\|v\|_{H^{l+1}\left(0, t_{n} ; L^{2}\right)}\right\}
$$

Lemma 3 Let $P_{h}$ and $P_{k}$ be the projections defined before, and let $u, v \in H^{1}\left(0, t_{n} ; H_{0}^{1}(\Omega)\right)$. Then, for any $(\psi, \phi) \in U_{h k}^{2}$, we have

$$
\begin{gathered}
\int_{0}^{t_{n}}\left[\left(\left(P_{h} v-v^{h k}\right)_{t}, \psi_{t}\right)+\left(\nabla\left(P_{k} P_{h} u-u^{h k}\right), \nabla \psi_{t}\right)\right] \mathrm{d} t \\
=\int_{0}^{t_{n}}\left[\left(\left(P_{h} v-v\right)_{t}, \psi_{t}\right)+\left(\nabla\left(P_{k} u-u\right), \nabla \psi_{t}\right)\right] \mathrm{d} t
\end{gathered}
$$

and

$$
\int_{0}^{t_{n}}\left[\left(\left(P_{k} P_{h} u-u^{h k}\right)_{t}, \phi_{t}\right)-\left(P_{h} v-v^{h k}, \phi_{t}\right)\right] \mathrm{d} t=0
$$

Proof From the definitions of $P_{h}$ and $P_{k}$ and the properties of the projections we get

$$
\begin{aligned}
& \int_{0}^{t_{n}}\left[\left(\left(P_{h} v-v^{h k}\right)_{t}, \psi_{t}\right)+\left(\nabla\left(P_{k} P_{h} u-u^{h k}\right), \nabla \psi_{t}\right)\right] \mathrm{d} t \\
& \quad=\int_{0}^{t_{n}}\left[\left(\left(P_{h} v-v\right)_{t}, \psi_{t}\right)+\left(\nabla\left(P_{k} P_{h} u-P_{h} u\right), \nabla \psi_{t}\right)\right] \mathrm{d} t
\end{aligned}
$$




$$
\begin{aligned}
& +\int_{0}^{t_{n}}\left[\left(\left(v-v^{h k}\right)_{t}, \psi_{t}\right)+\left(\nabla\left(P_{h} u-u^{h k}\right), \nabla \psi_{t}\right)\right] \mathrm{d} t \\
= & \int_{0}^{t_{n}}\left[\left(\left(P_{h} v-v\right)_{t}, \psi_{t}\right)+\left(\nabla\left(P_{k} u-u\right), \nabla \psi_{t}\right)\right] \mathrm{d} t \\
& +\int_{0}^{t_{n}}\left[\left(\left(v-v^{h k}\right)_{t}, \psi_{t}\right)+\left(\nabla\left(u-u^{h k}\right), \nabla \psi_{t}\right)\right] \mathrm{d} t .
\end{aligned}
$$

In addition,

$$
\begin{gathered}
\int_{0}^{t_{n}}\left[\left(\left(P_{k} P_{h} u-u^{h k}\right)_{t}, \phi_{t}\right)-\left(\left(P_{h} v-v^{h k}\right), \phi_{t}\right)\right] \mathrm{d} t \\
=\int_{0}^{t_{n}}\left[\left(\left(P_{k} P_{h} u-u\right)_{t}, \phi_{t}\right)-\left(P_{h} v-v, \phi_{t}\right)\right] \mathrm{d} t \\
\quad+\int_{0}^{t_{n}}\left[\left(\left(u-u^{h k}\right)_{t}, \phi_{t}\right)-\left(v-v^{h k}, \phi_{t}\right)\right] \mathrm{d} t \\
=\int_{0}^{t_{n}}\left[\left(\left(P_{h} u-u\right)_{t}, \phi_{t}\right)-\left(P_{h} v-v, \phi_{t}\right)\right] \mathrm{d} t \\
\quad+\int_{0}^{t_{n}}\left[\left(\left(u-u^{h k}\right)_{t}, \phi_{t}\right)-\left(v-v^{h k}, \phi_{t}\right)\right] \mathrm{d} t .
\end{gathered}
$$

Because $(u, v)$ and $\left(u^{h k}, v^{h k}\right)$ are solution pairs of (2.2)-(2.4) and (2.5)-(2.7), respectively, combining (3.14)-(3.15) with the fact $v=u_{t}$ finishes the proof of Lemma 3.

In order to continue the theoretical analysis, the following Gronwall lemma needs to be recalled. In the sequel, it will be used frequently.

Lemma 4 (Gronwall Lemma) Suppose $y(s), g(s), h(s)$ are three nonnegative locally integrable functions on the interval $[0, \infty)$ and that, for any given $t_{0}$ and all $t \geq t_{0}$, the following inequality holds:

$$
g(t)+W(t) \leq C+\int_{t_{0}}^{t} y(\tau) \mathrm{d} \tau+\int_{t_{0}}^{t} g(\tau) h(\tau) \mathrm{d} \tau
$$

Then,

$$
g(t)+W(t) \leq\left(C+\int_{t_{0}}^{t} y(\tau) \mathrm{d} \tau\right) \exp \left(\int_{t_{0}}^{t} h(\tau) \mathrm{d} \tau\right)
$$

where $W(t)$ is a nonnegative function, and $C \geq 0$ represents a positive constant that does not depend on $k$ and $h$.

The results on the convergence of the numerical solutions of (2.5)-(2.7) are given in the following theorems and corollary.

Theorem 2 Let $(u(x, t), v(x, t))$ be a solution pair of (2.2)-(2.4), and let $\left(u^{h k}(x, t), v^{h k}(x, t)\right)$ satisfy (2.5)-(2.7). Then the following error estimates hold: 
(1) Let $\nabla u \in H^{m+1}(\Omega)$ for $0 \leq t \leq T, \Delta u \in H^{l+1}\left(0, T ; L^{2}(\Omega)\right), v_{t} \in H^{1}\left(0, T ; H^{m+1}(\Omega)\right)$, and $v_{t t} \in L^{2}\left(0, T ; H^{m+1}(\Omega)\right)$. Then

$$
\begin{aligned}
& \left\|\left(u\left(t_{n}\right)-u^{h k}\left(t_{n}\right)\right)\right\|_{1} \\
& \leq C\left\{h^{m}\left(\left\|\nabla u\left(t_{n}\right)\right\|_{m+1}+\left\|v_{t t}\right\|_{L^{2}\left(0, t_{n} ; H^{m+1}\right)}+\sup _{0 \leq t \leq t_{n}}\left\|v_{t}\right\|_{m+1}\right)\right. \\
& \left.\quad+k^{l}\|\Delta u\|_{H^{l+1}\left(0, t_{n} ; L^{2}\right)}\right\}, \quad n=1,2, \ldots, N .
\end{aligned}
$$

(2) Let $v \in H^{m+1}(\Omega)$ for $0 \leq t \leq T, \Delta u \in H^{l+1}\left(0, T ; L^{2}(\Omega)\right), v_{t} \in H^{1}\left(0, T ; H^{m+1}(\Omega)\right)$, and $v_{t t} \in L^{2}\left(0, T ; H^{m+1}(\Omega)\right)$. Then

$$
\begin{aligned}
& \left\|\left(v\left(t_{n}\right)-v^{h k}\left(t_{n}\right)\right)\right\| \\
& \leq C \\
& \quad C\left\{h^{m+1}\left(\left\|v\left(t_{n}\right)\right\|_{m+1}+\left\|v_{t t}\right\|_{L^{2}\left(0, t_{n} ; H^{m+1}\right)}+\sup _{0 \leq t \leq t_{n}}\left\|v_{t}\right\|_{m+1}\right)\right. \\
& \left.\quad+k^{l}\|\Delta u\|_{H^{l+1}\left(0, t_{n} ; L^{2}\right)}\right\}, \quad n=1,2, \ldots, N .
\end{aligned}
$$

Proof Taking $(\psi, \phi)=\left(P_{k} P_{h} u-u^{h k}, P_{h} v-v^{h k}\right)$ in (3.12) and (3.13), we obtain

$$
\begin{gathered}
\int_{0}^{t_{h}}\left[\left(P_{h} v-v^{h k},\left(P_{h} v-v^{h k}\right)_{t}\right)+\left(\nabla\left(P_{k} P_{h} u-u^{h k}\right), \nabla\left(P_{k} P_{h} u-u^{h k}\right)_{t}\right)\right] \mathrm{d} t \\
=\int_{0}^{t_{h}}\left[\left(\left(P_{h} v-v\right)_{t},\left(P_{k} P_{h} u-u^{h k}\right)_{t}\right)+\left(\Delta\left(P_{k} u-u\right),\left(P_{k} P_{h} u-u^{h k}\right)_{t}\right)\right] \mathrm{d} t .
\end{gathered}
$$

Noting that $\left(P_{h} v(0), P_{h} u(0)\right)=\left(v^{h k}(0), u^{h k}(0)\right)$ and $P_{k} w\left(t_{n}\right)=w\left(t_{n}\right)\left(\forall w \in H^{1}(0, T)\right)$ and using integration by parts to the right side of (3.20), we have

$$
\begin{aligned}
& \frac{1}{2}\left\|P_{h} v\left(t_{n}\right)-v^{h k}\left(t_{n}\right)\right\|_{0}^{2}+\frac{1}{2}\left\|\nabla\left(P_{h} P_{k} u\left(t_{n}\right)-u^{h k}\left(t_{n}\right)\right)\right\|_{0}^{2} \\
& \leq \\
& \quad\left(\left(P_{h} v-v\right)_{t}\left(t_{n}\right),\left(P_{k} P_{h} u-u^{h k}\right)\left(t_{n}\right)\right) \\
& \quad+\int_{0}^{t_{n}}\left(P_{k} P_{h} u-u^{h k},\left(P_{h} v-v\right)_{t t}\right) \mathrm{d} t \\
& \quad+\int_{0}^{t_{n}}\left(P_{k} P_{h} u-u^{h k}, \Delta\left(P_{k} u-u\right)_{t}\right) \mathrm{d} t .
\end{aligned}
$$

Using the Hölder and Cauchy inequalities, we have

$$
\begin{aligned}
& \left\|P_{h} v\left(t_{n}\right)-v^{h k}\left(t_{n}\right)\right\|_{0}^{2}+\left\|\nabla\left(P_{h} P_{k} u\left(t_{n}\right)-u^{h k}\left(t_{n}\right)\right)\right\|_{0}^{2} \\
& \leq c\left\|\left(P_{h} v-v\right)_{t}\left(t_{n}\right)\right\|^{2}+c \int_{0}^{t_{n}}\left\|\left(P_{h} v-v\right)_{t t}\right\|^{2} \mathrm{~d} t \\
& \quad+c \int_{0}^{t_{n}}\left\|\Delta\left(P_{k} u-u\right)_{t}\right\|^{2} \mathrm{~d} t+\int_{0}^{t_{n}}\left\|\nabla\left(P_{k} P_{h} u-u^{h k}\right)\right\| \mathrm{d} t .
\end{aligned}
$$

Applying the Gronwall lemma to (3.22) yields

$$
\begin{aligned}
& \left\|P_{h} v\left(t_{n}\right)-v^{h k}\left(t_{n}\right)\right\|_{0}^{2}+\left\|\nabla\left(P_{h} P_{k} u\left(t_{n}\right)-u^{h k}\left(t_{n}\right)\right)\right\|_{0}^{2} \\
& \quad \leq c \max _{0 \leq t \leq T}\left\|\left(P_{h} v-v\right)_{t}\right\|^{2}+c \int_{0}^{t_{n}}\left\|\left(P_{h} v-v\right)_{t t}\right\|^{2} \mathrm{~d} t+c \int_{0}^{t_{n}}\left\|\Delta\left(P_{k} u-u\right)_{t}\right\|^{2} \mathrm{~d} t .
\end{aligned}
$$


By the triangle inequality and (3.23), noting that $P_{k} P_{h} u\left(t_{n}\right)=P_{h} u\left(t_{n}\right)$, we see that

$$
\begin{aligned}
\left\|\nabla\left(u\left(t_{n}\right)-u^{h k}\left(t_{n}\right)\right)\right\| \leq & \left\|\nabla\left(u\left(t_{n}\right)-P_{h} u\left(t_{n}\right)\right)\right\|+\left\|\nabla\left(P_{k} P_{h} u\left(t_{n}\right)-u^{h k}\left(t_{n}\right)\right)\right\| \\
\leq & c \sup _{0 \leq t \leq T}\left\|\left(P_{h} v-v\right)_{t}\right\|+c\left\|\left(P_{h} v-v\right)_{t t}\right\|_{L^{2}\left(0, t_{n} ; L^{2}\right)} \\
& +c\left\|\Delta\left(P_{k} u-u\right)_{t}\right\|_{L^{2}\left(0, t_{n} ; L^{2}\right)}+\left\|\nabla\left(u\left(t_{n}\right)-P_{h} u\left(t_{n}\right)\right)\right\| .
\end{aligned}
$$

Then (3.18) follows from (3.24) and Lemma 2. Again, using the triangle inequality and (3.23), we obtain

$$
\begin{aligned}
\left\|v\left(t_{n}\right)-v^{h k}\left(t_{n}\right)\right\| \leq & \left\|v\left(t_{n}\right)-P_{h} v\left(t_{n}\right)\right\|+\left\|P_{h} v\left(t_{n}\right)-v^{h k}\left(t_{n}\right)\right\| \\
\leq & c \sup _{0 \leq t \leq T}\left\|\left(P_{h} v-v\right)_{t}\right\|+c\left\|\left(P_{h} v-v\right)_{t t}\right\|_{L^{2}\left(0, t_{n} ; L^{2}\right)} \\
& +c\left\|\Delta\left(P_{k} u-u\right)_{t}\right\|_{L^{2}\left(0, t_{n} ; L^{2}\right)}+\left\|v\left(t_{n}\right)-P_{h} v\left(t_{n}\right)\right\| .
\end{aligned}
$$

By (3.25) and Lemma 2 we finish the proof of part 2 of Theorem 2.

We also give the energy norm estimate in the following corollary.

Corollary 1 Suppose that the conditions of the Theorem 2 hold. Then

$$
\begin{aligned}
& \left\|u\left(t_{n}\right)-u^{h k}\left(t_{n}\right)\right\|_{1}+\left\|v\left(t_{n}\right)-v^{h k}\left(t_{n}\right)\right\| \\
& \leq C\left\{h^{m}\left(\left\|\nabla u\left(t_{n}\right)\right\|_{m+1}+\left\|v\left(t_{n}\right)\right\|_{m+1}+\left\|v_{t t}\right\|_{L^{2}\left(0, t_{n} ; H^{m+1}\right)}+\sup _{0 \leq t \leq t_{n}}\left\|v_{t}\right\|_{m+1}\right)\right. \\
& \left.\quad+k^{l}\|\Delta u\|_{H^{l+1}\left(0, t_{n} ; L^{2}\right)}\right\}, \quad n=1,2, \ldots, N .
\end{aligned}
$$

Proof The corollary is directly demonstrated via the results (3.18) and (3.19) of Theorem 2 .

Theorem 3 Suppose that solutions $u$ and $v$ to (2.2)-(2.4) are smooth enough so that $u \in H^{m+1}(\Omega)$ for $0 \leq t \leq T, \Delta u \in H^{l+1}\left(0, T ; L^{2}(\Omega)\right), v_{t} \in H^{1}\left(0, T ; H^{m+1}(\Omega)\right)$, and $v_{t t} \in$ $L^{2}\left(0, T ; H^{m+1}(\Omega)\right)$. Then we have

$$
\begin{aligned}
\left\|\left(u\left(t_{n}\right)-u^{h k}\left(t_{n}\right)\right)\right\| \leq & C\left\{h^{m+1}\left(\left\|u\left(t_{n}\right)\right\|_{m+1}+\left\|v_{t t}\right\|_{L^{2}\left(0, t_{n} ; H^{m+1}\right)}+\sup _{0 \leq t \leq T}\left\|v_{t}\right\|_{m+1}\right)\right. \\
& \left.+k^{l}\|\Delta u\|_{H^{l+1}\left(0, t_{n} ; L^{2}\right)}\right\}, \quad n=1,2, \ldots, N .
\end{aligned}
$$

Proof Setting $(\psi, \varphi)=\left(A_{h}\left(P_{k} P_{h} u-u^{h k}\right), A_{h}\left(P_{h} v-v^{h k}\right)\right)$ in (3.12) and (3.13) and applying the definition and symmetry property of $A_{h}$, we obtain

$$
\begin{aligned}
& \int_{0}^{t_{h}}\left[\left(\left(P_{h} v-v^{h k}\right), A_{h}\left(P_{h} v-v^{h k}\right)_{t}\right)+\left(\left(P_{k} P_{h} u-u^{h k}\right),\left(P_{k} P_{h} u-u^{h k}\right)_{t}\right)\right] \mathrm{d} t \\
& =\int_{0}^{t_{n}}\left(\left(P_{h} v-v\right)_{t}, A_{h}\left(P_{k} P_{h} u-u^{h k}\right)_{t}\right) \mathrm{d} t \\
& \quad+\int_{0}^{t_{h}}\left(\nabla\left(P_{k} u-u\right), \nabla A_{h}\left(P_{k} P_{h} u-u^{h k}\right)_{t}\right) \mathrm{d} t .
\end{aligned}
$$


From the initial condition $\left(P_{h} v(0), P_{h} u(0)\right)=\left(v^{h k}(0), u^{h k}(0)\right)$ and $P_{k} w\left(t_{n}\right)=w\left(t_{n}\right)(\forall w \in$ $\left.H^{1}(0, T)\right)$, using integration by parts to the right side of (3.28), we see that

$$
\begin{gathered}
\frac{1}{2}\left\|A_{h}^{1 / 2}\left(P_{h} v-v^{h k}\right)\left(t_{n}\right)\right\|_{0}^{2}+\frac{1}{2}\left\|\left(P_{k} P_{h} u-u^{h k}\right)\left(t_{n}\right)\right\|_{0}^{2} \\
\leq\left(A_{h}\left(P_{h} v-v\right)_{t}\left(t_{n}\right),\left(P_{k} P_{h} u-u^{h k}\right)\left(t_{n}\right)\right) \\
\quad+\int_{0}^{t_{n}}\left(P_{k} P_{h} u-u^{h k}, A_{h}\left(P_{h} v-v\right)_{t t}\right) \mathrm{d} t \\
\quad+\int_{0}^{t_{n}}\left(P_{k} P_{h} u-u^{h k}, A_{h}\left(P_{k} \Delta u-\Delta u\right)_{t}\right) \mathrm{d} t .
\end{gathered}
$$

Further, using the Hölder and Cauchy inequalities, we have that

$$
\begin{aligned}
& \left\|A_{h}^{1 / 2}\left(P_{h} v-v^{h k}\right)\left(t_{n}\right)\right\|^{2}+\left\|P_{k} P_{h} u\left(t_{n}\right)-u^{h k}\left(t_{n}\right)\right\|^{2} \\
& \leq c \sup _{0 \leq t \leq T}\left\|A_{h}\left(P_{h} v-v\right)_{t}\right\|^{2}+c \int_{0}^{t_{n}}\left\|A_{h}\left(P_{h} v-v\right)_{t t}\right\|^{2} \mathrm{~d} t \\
& \quad+c \int_{0}^{t_{n}}\left\|A_{h}\left(P_{k} \Delta u-\Delta u\right)_{t}\right\|^{2} \mathrm{~d} t+\int_{0}^{t_{n}}\left\|\left(P_{k} P_{h} u-u^{h k}\right)\right\|^{2} \mathrm{~d} t .
\end{aligned}
$$

Applying the Gronwall lemma to (3.30) yields

$$
\begin{aligned}
& \left\|A_{h}^{1 / 2}\left(P_{h} v-v^{h k}\right)\left(t_{n}\right)\right\|^{2}+\left\|P_{k} P_{h} u\left(t_{n}\right)-u^{h k}\left(t_{n}\right)\right\|^{2} \\
& \leq c \sup _{0 \leq t \leq T}\left\|A_{h}\left(P_{h} v-v\right)_{t}\right\|^{2}+c \int_{0}^{t_{n}}\left\|A_{h}\left(P_{h} v-v\right)_{t t}\right\|^{2} \mathrm{~d} t \\
& \quad+c \int_{0}^{t_{n}}\left\|A_{h}\left(\Delta u-P_{k} \Delta u\right)_{t}\right\|^{2} \mathrm{~d} t .
\end{aligned}
$$

Therefore, applying the triangle inequality to (3.31), we get that

$$
\begin{aligned}
& \left\|u\left(t_{n}\right)-u^{h k}\left(t_{n}\right)\right\| \\
& \leq\left\|u\left(t_{n}\right)-P_{h} u\left(t_{n}\right)\right\|+\left\|P_{k} P_{h} u\left(t_{n}\right)-u^{h k}\left(t_{n}\right)\right\| \\
& \leq c \sup _{0 \leq t \leq T}\left\|A_{h}\left(P_{h} v-v\right)_{t}\right\|+c\left\|A_{h}\left(P_{h} v-v\right)_{t t}\right\|_{L^{2}\left(0, t_{n} ; L^{2}\right)} \\
& \quad+c\left\|A_{h}\left(P_{k} \Delta u-\Delta u\right)_{t}\right\|_{L^{2}\left(0, t_{n} ; L^{2}\right)}+\left\|u\left(t_{n}\right)-P_{h} u\left(t_{n}\right)\right\| .
\end{aligned}
$$

Inequality (3.27) now directly follows from (3.32), the boundedness of $A_{h}$, and Lemma 2.

Theorem 4 Let $\nabla v \in H^{m+1}(\Omega)$ for $0 \leq t \leq T, \Delta u \in H^{l+1}\left(0, T ; L^{2}(\Omega)\right), v_{t} \in H^{1}(0, T$; $\left.H^{m+1}(\Omega)\right)$, and $v_{t t} \in L^{2}\left(0, T ; H^{m+1}(\Omega)\right)$. Then

$$
\begin{aligned}
\left\|\left(v\left(t_{n}\right)-v^{h k}\left(t_{n}\right)\right)\right\|_{1} \leq & C\left\{h^{m}\left(\left\|\nabla v\left(t_{n}\right)\right\|_{m+1}+\left\|v_{t t}\right\|_{L^{2}\left(0, t_{n} ; H^{m+1}\right)}+\sup _{0 \leq t \leq t_{n}}\left\|v_{t}\right\|_{m+1}\right)\right. \\
& \left.+k^{l}\|\Delta u\|_{H^{l+1}\left(0, t_{n} ; L^{2}\right)}\right\}, \quad n=1,2, \ldots, N .
\end{aligned}
$$


Proof We first introduce the discrete space-time operator $T_{h}: L^{2}\left(0, T ; H_{0}^{1}(\Omega)\right) \rightarrow S_{h m}(\Omega) \times$ $L^{2}(0, T)$ satisfying

$$
\int_{0}^{T}\left(T_{h} \chi, \phi\right) \mathrm{d} t=\int_{0}^{T}(\nabla \chi, \nabla \phi) \mathrm{d} t, \quad \forall \phi \in S_{h m}(\Omega) \times L^{2}(0, T) .
$$

Taking $(\psi, \varphi)=\left(T_{h}\left(P_{k} P_{h} u-u^{h k}\right), T_{h}\left(P_{h} v-v^{h k}\right)\right)$ in (3.12) and (3.13) and applying the definition of $T_{h}$, we obtain

$$
\begin{aligned}
\int_{0}^{t_{n}} & {\left[\left(\nabla\left(P_{h} v-v^{h k}\right), \nabla\left(P_{h} v-v^{h k}\right)_{t}\right)\right.} \\
& \left.+\left(T_{h}\left(P_{k} P_{h} u-u^{h k}\right), T_{h}\left(P_{k} P_{h} u-u^{h k}\right)_{t}\right)\right] \mathrm{d} t \\
= & \int_{0}^{t_{n}}\left(\left(P_{h} v-v\right)_{t}, T_{h}\left(P_{k} P_{h} u-u^{h k}\right)_{t}\right) \mathrm{d} t \\
& -\int_{0}^{t_{n}}\left(\Delta\left(P_{k} u-u\right), T_{h}\left(P_{k} P_{h} u-u^{h k}\right)_{t}\right) \mathrm{d} t .
\end{aligned}
$$

Further,

$$
\begin{aligned}
& \left\|\nabla\left(P_{h} v-v^{h k}\right)\left(t_{n}\right)\right\|^{2}+\left\|T_{h}\left(P_{k} P_{h} u-u^{h k}\right)\left(t_{n}\right)\right\|^{2} \\
& \leq c \sup _{0 \leq t \leq T}\left\|\left(P_{h} v-v\right)_{t}\right\|^{2}+c \int_{0}^{t_{n}}\left\|\left(P_{h} v-v\right)_{t t}\right\|^{2} \mathrm{~d} t \\
& \quad+c \int_{0}^{t_{n}}\left\|\left(P_{k} \Delta u-\Delta u\right)_{t}\right\|^{2} \mathrm{~d} t+\int_{0}^{t_{n}}\left\|T_{h}\left(P_{k} P_{h} u-u^{h k}\right)\right\|^{2} \mathrm{~d} t .
\end{aligned}
$$

Then applying the Gronwall lemma to (3.36) yields

$$
\begin{aligned}
& \left\|\nabla\left(P_{h} v-v^{h k}\right)\left(t_{n}\right)\right\|^{2}+\left\|T_{h}\left(P_{k} P_{h} u-u^{h k}\right)\left(t_{n}\right)\right\|^{2} \\
& \leq c \sup _{0 \leq t \leq T}\left\|\left(P_{h} v-v\right)_{t}\right\|^{2}+c \int_{0}^{t_{n}}\left\|\left(P_{h} v-v\right)_{t t}\right\|^{2} \mathrm{~d} t \\
& \quad+c \int_{0}^{t_{n}}\left\|\left(P_{k} \Delta u-\Delta u\right)_{t}\right\|^{2} \mathrm{~d} t .
\end{aligned}
$$

Therefore,

$$
\begin{aligned}
& \left\|\nabla\left(v-v^{h k}\right)\left(t_{n}\right)\right\| \\
& \leq\left\|\nabla\left(v-P_{h} v\right)\left(t_{n}\right)\right\|+\left\|\nabla\left(P_{h} v-v^{h k}\right)\left(t_{n}\right)\right\| \\
& \leq c \sup _{0 \leq t \leq T}\left\|\left(P_{h} v-v\right)_{t}\right\|+c\left\|\left(P_{h} v-v\right)_{t t}\right\|_{L^{2}\left(0, t_{n} ; L^{2}\right)} \\
& \quad+c\left\|\left(P_{k} \Delta u-\Delta u\right)_{t}\right\|_{L^{2}\left(0, t_{n} ; L^{2}\right)}+\left\|\nabla\left(v-P_{h} v\right)\left(t_{n}\right)\right\| .
\end{aligned}
$$

In view of (3.38), Lemma 2 completes the proof for Theorem 4.

\section{Numerical example}

In this part, a numerical example is provided to validate the efficiency and feasibility of the STCG method presented in this paper. We discretize (1.1) on the unit spatial domain 
Figure 1 A partition of the domain with $h=1 / 16$

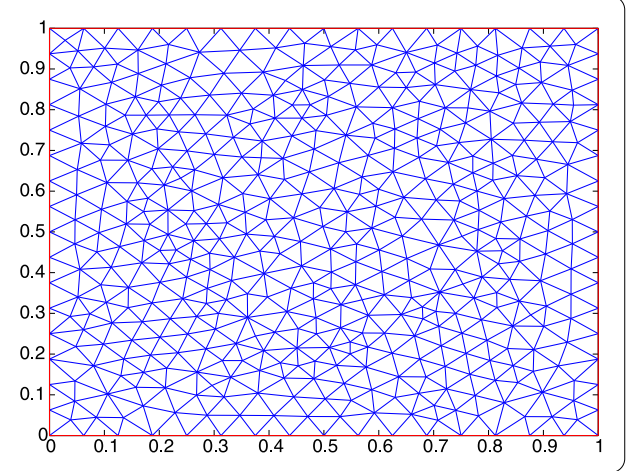

Table 1 The errors and convergence rates of $u-u^{h k}$ in the $L^{2}$ and $H^{1}$ norms concerning $h$ at $t=1$

\begin{tabular}{lllll}
\hline $\boldsymbol{h}$ & Error in $\boldsymbol{L}^{\mathbf{2}}$ & Rate & Error in $\boldsymbol{H}^{\mathbf{1}}$ & Rate \\
\hline $1 / 8$ & $4.8975 \mathrm{e}-2$ & & $1.6055 \mathrm{e}-0$ & \\
$1 / 16$ & $1.2272 \mathrm{e}-2$ & 1.9967 & $8.0820 \mathrm{e}-1$ & 0.9902 \\
$1 / 32$ & $3.0747 \mathrm{e}-3$ & 1.9968 & $4.0534 \mathrm{e}-1$ & 0.9956 \\
$1 / 64$ & $7.6265 \mathrm{e}-4$ & 2.0113 & $2.0206 \mathrm{e}-2$ & 1.0043 \\
\hline
\end{tabular}

Table 2 The errors and convergence rates of $v-v^{h k}$ in the $L^{2}$ and $H^{1}$ norms concerning $h$ at $t=1$

\begin{tabular}{lllll}
\hline $\boldsymbol{h}$ & Error in $\boldsymbol{L}^{\mathbf{2}}$ & Rate & Error in $\boldsymbol{H}^{\mathbf{1}}$ & Rate \\
\hline $1 / 8$ & $2.4889 \mathrm{e}-2$ & & $8.0276 \mathrm{e}-1$ & \\
$1 / 16$ & $6.2303 \mathrm{e}-3$ & 1.9981 & $4.0410 \mathrm{e}-1$ & 0.9903 \\
$1 / 32$ & $1.4268 \mathrm{e}-3$ & 2.1266 & $2.0268 \mathrm{e}-1$ & 0.9955 \\
$1 / 64$ & $2.3105 \mathrm{e}-4$ & 2.6265 & $1.0109 \mathrm{e}-1$ & 1.0036 \\
\hline
\end{tabular}

Table 3 The errors and convergence rates of $u-u^{h k}$ in the $L^{2}\left(L^{2}\right)$ and $L^{2}\left(H^{1}\right)$ norms concerning $h$

\begin{tabular}{lllll}
\hline $\boldsymbol{h}$ & Error in $\boldsymbol{L}^{\mathbf{2}} \mathbf{L}^{\mathbf{2}} \mathbf{)}$ & Rate & Error in $\left.\boldsymbol{L}^{\mathbf{2}} \boldsymbol{H}^{\mathbf{1}}\right)$ & Rate \\
\hline $1 / 8$ & $6.4243 \mathrm{e}-2$ & & $2.1045 \mathrm{e}-0$ & \\
$1 / 16$ & $1.6078 \mathrm{e}-2$ & 1.9985 & $1.0594 \mathrm{e}-0$ & 0.9902 \\
$1 / 32$ & $4.0188 \mathrm{e}-3$ & 2.0003 & $5.3134 \mathrm{e}-1$ & 0.9956 \\
$1 / 64$ & $9.8849 \mathrm{e}-4$ & 2.0235 & $2.6487 \mathrm{e}-1$ & 1.0043 \\
\hline
\end{tabular}

$\Omega=[0,1]^{2}$ and the time span $I=[0,1]$. The exact solutions $u=e^{-\frac{t}{2}} \sin (2 \pi x) \sin (4 \pi y)$ and $\nu=-\frac{1}{2} e^{-\frac{t}{2}} \sin (2 \pi x) \sin (4 \pi y)$ are established by (2.1) if $f=\left(1+20 \pi^{2}\right) e^{-\frac{t}{2}} \sin (2 \pi x) \sin (4 \pi y)$, $u_{0}=\sin (2 \pi x) \sin (4 \pi y)$, and $v_{0}=-\frac{1}{2} \sin (2 \pi x) \sin (4 \pi y)$. We set discrete initial values $\left(u^{h k}(0), v^{h k}(0)\right)=\left(P_{h} u_{0}, P_{h} v_{0}\right)$. We also provide the errors and orders of convergence in the $L^{2}\left(L^{2}\right)$ and $L^{2}\left(H^{1}\right)$ norms for $u-u^{h k}$ and $v-v^{h k}$ concerning $h$ and $k$. All the experiments are simulated on unstructured meshes (see Figure 1) and computed from $t=0$ to $t=1$.

In this example, we set a linear polynomial regarding space variables and a quadratic polynomial regarding time variable, that is, $m=1, l=2$. Firstly, we investigate the orders of convergence concerning $h$. To achieve this goal, we fix the time step $k=0.001$ and set the mesh size $h=\frac{1}{8}, \frac{1}{16}, \frac{1}{32}, \frac{1}{64}$, respectively. Table 1 , Table 2, Table 3, and Table 4 indicate that the first-order accuracy in the $L^{\infty}\left(H^{1}\right)$ and $H^{1}$ norms and the second-order accuracy in the $L^{\infty}\left(L^{2}\right)$ and $L^{2}$ norms are obtained, in accordance with theoretical analysis. Moreover, we 
Table 4 The errors and convergence rates of $v-v^{h k}$ in the $L^{2}\left(L^{2}\right)$ and $L^{2}\left(H^{1}\right)$ norms concerning $h$

\begin{tabular}{lllll}
\hline $\boldsymbol{h}$ & Error in $\left.\boldsymbol{L}^{\mathbf{2}} \mathbf{L}^{\mathbf{2}}\right)$ & Rate & Error in $\boldsymbol{L}^{\mathbf{2}} \boldsymbol{H}^{\mathbf{1}} \mathbf{)}$ & Rate \\
\hline $1 / 8$ & $3.2219 \mathrm{e}-2$ & & $1.0523 \mathrm{e}-0$ & \\
$1 / 16$ & $8.0528 \mathrm{e}-3$ & 2.0004 & $5.2971 \mathrm{e}-1$ & 0.9903 \\
$1 / 32$ & $2.0065 \mathrm{e}-3$ & 2.0048 & $2.6567 \mathrm{e}-1$ & 0.9955 \\
$1 / 64$ & $5.0324 \mathrm{e}-4$ & 1.9953 & $1.3247 \mathrm{e}-1$ & 1.0040 \\
\hline
\end{tabular}

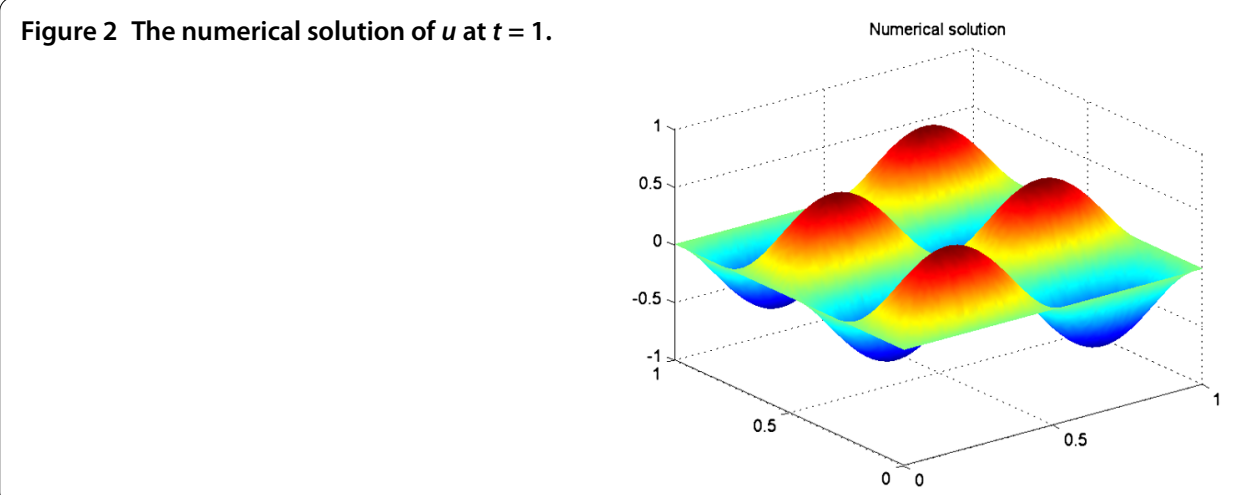

Figure 3 The exact solution of $u$ at $t=1$.

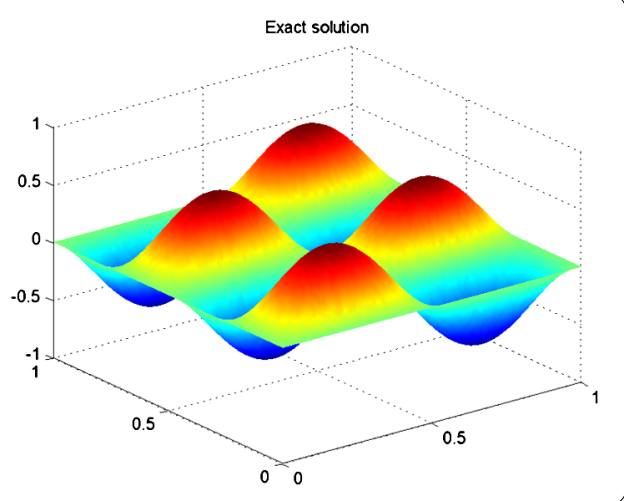

provide the plots to numerical solutions and exact solutions for $u$ in Figure 2 and Figure 3 and for $v$ in Figure 4 and Figure 5 with $h=1 / 64$, both of which show that the numerical solutions are very close to the exact solutions.

Next, we analyze the orders of convergence with respect to $k$. Because we take $l=2$ in this example, the errors in the $L^{2}\left(L^{2}\right), L^{2}\left(H^{1}\right), L^{2}$, and $H^{1}$ norms theoretically should have the third-order accuracy concerning $k$. Thus, in order to study the orders of convergence in the $L^{\infty}\left(L^{2}\right), L^{2}$ and $L^{\infty}\left(H^{1}\right), H^{1}$ norms concerning $k$, we take $h=O\left(k^{3 / 2}\right)$ and $h=O\left(k^{3}\right)$, respectively. In this case, the errors are only functions of time step $k$. Table 5 , Table 6 , Table 7, and Table 8 show that the orders of convergence for $u-u^{h k}$ and $v-v^{h k}$ in these norms near third-order accuracy concerning $k$, respectively, which are one order accuracy higher than the theoretical findings.

\section{Conclusions and perspectives}

In this paper, we propose a new theoretical analysis on the STCG method for wave equation. We prove the existence and uniqueness to the STCG solutions and get the optimal 
Figure 4 The numerical solution of $v$ at $t=1$.

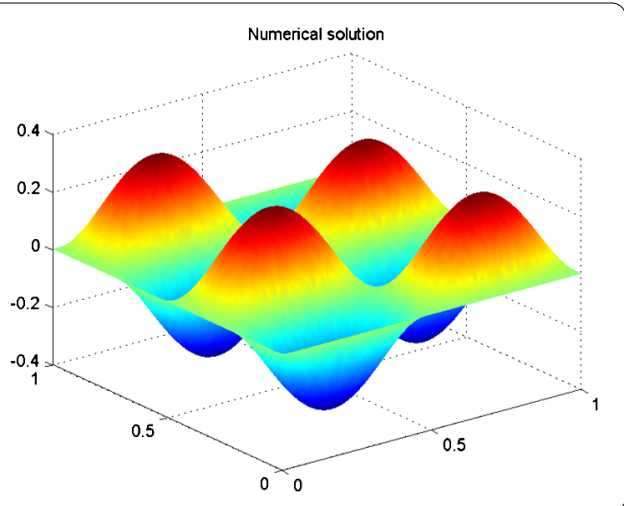

Figure 5 The exact solution of $v$ at $t=1$.

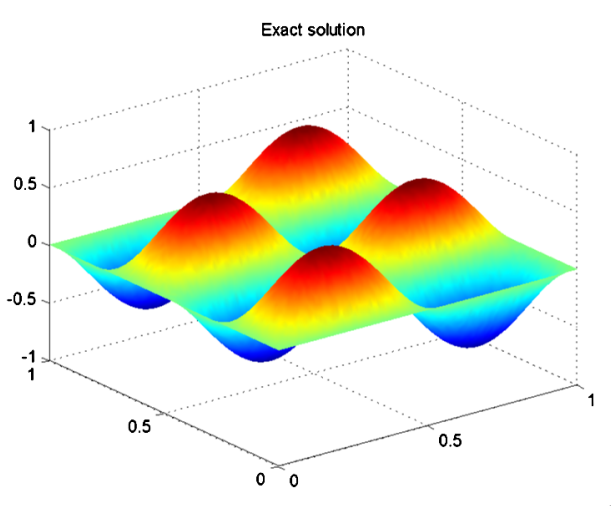

Table 5 The errors and convergence rates of $u-u^{h k}$ in the $L^{2}$ and $H^{1}$ norms concerning $k$ at $t=1$

\begin{tabular}{llllll}
\hline $\boldsymbol{( h , k )}$ & Error in $\boldsymbol{L}^{\mathbf{2}}$ & Rate & $(\boldsymbol{h}, \boldsymbol{k})$ & Error in $\boldsymbol{H}^{\mathbf{1}}$ & Rate \\
\hline$(0.125,1 / 4)$ & $2.4620 \mathrm{e}-2$ & & $(1 / 8,1 / 2)$ & $8.0728 \mathrm{e}-1$ & \\
$(0.0442,1 / 8)$ & $3.1414 \mathrm{e}-3$ & 2.9703 & $(1 / 27,1 / 3)$ & $2.4231 \mathrm{e}-1$ & 2.9681 \\
$(0.0156,1 / 16)$ & $3.9093 \mathrm{e}-4$ & 3.0064 & $(1 / 64,1 / 4)$ & $1.0114 \mathrm{e}-1$ & 3.0372 \\
\hline
\end{tabular}

Table 6 The errors and convergence rates of $v-v^{h k}$ in the $L^{2}$ and $H^{1}$ norms concerning $k$ at $t=1$

\begin{tabular}{llllll}
\hline$(\boldsymbol{h}, \boldsymbol{k})$ & Error in $\boldsymbol{L}^{\mathbf{2}}$ & Rate & $(\boldsymbol{h}, \boldsymbol{k})$ & Error in $\boldsymbol{H}^{\mathbf{1}}$ & Rate \\
\hline$(0.125,1 / 4)$ & $1.2556 \mathrm{e}-2$ & & $(1 / 8,1 / 2)$ & $4.0417 \mathrm{e}-1$ & \\
$(0.0442,1 / 8)$ & $1.5500 \mathrm{e}-3$ & 3.0181 & $(1 / 27,1 / 3)$ & $1.2116 \mathrm{e}-1$ & 2.9712 \\
$(0.0156,1 / 16)$ & $1.1918 \mathrm{e}-4$ & 3.7010 & $(1 / 64,1 / 4)$ & $5.0577 \mathrm{e}-2$ & 3.0367 \\
\hline
\end{tabular}

Table 7 The errors and convergence rates of $u-u^{h k}$ in the $L^{2}\left(L^{2}\right)$ and $L^{2}\left(H^{1}\right)$ norms concerning $k$

\begin{tabular}{llllll}
\hline $\boldsymbol{( h , k )}$ & Error in $\left.\mathbf{L}^{\mathbf{2}} \boldsymbol{L}^{\mathbf{2}}\right)$ & Rate & $(\boldsymbol{h}, \boldsymbol{k})$ & Error in $\mathbf{L}^{\mathbf{2}}\left(\boldsymbol{H}^{\mathbf{1}}\right)$ & Rate \\
\hline$(0.125,1 / 4)$ & $3.2246 \mathrm{e}-2$ & & $(1 / 8,1 / 2)$ & $1.0582 \mathrm{e}-0$ & \\
$(0.0442,1 / 8)$ & $4.1121 \mathrm{e}-3$ & 2.9712 & $(1 / 27,1 / 3)$ & $3.1763 \mathrm{e}-1$ & 2.9681 \\
$(0.0156,1 / 16)$ & $5.0685 \mathrm{e}-4$ & 3.0203 & $(1 / 64,1 / 4)$ & $1.3257 \mathrm{e}-1$ & 3.0372 \\
\hline
\end{tabular}

Table 8 The errors and convergence rates of $v-v^{h k}$ in the $L^{2}\left(L^{2}\right)$ and $L^{2}\left(H^{1}\right)$ norms concerning $k$

\begin{tabular}{llllll}
\hline $\boldsymbol{( h , k )}$ & Error in $\left.\mathbf{L}^{\mathbf{2}} \boldsymbol{L}^{\mathbf{2}}\right)$ & Rate & $(\boldsymbol{h}, \boldsymbol{k})$ & Error in $\mathbf{L}^{\mathbf{2}}\left(\boldsymbol{H}^{\mathbf{1}}\right)$ & Rate \\
\hline$(0.125,1 / 4)$ & $1.6141 \mathrm{e}-2$ & & $(1 / 8,1 / 2)$ & $5.2912 \mathrm{e}-1$ & \\
$(0.0442,1 / 8)$ & $2.0566 \mathrm{e}-3$ & 2.9724 & $(1 / 27,1 / 3)$ & $1.5882 \mathrm{e}-1$ & 2.9681 \\
$(0.0156,1 / 16)$ & $2.5788 \mathrm{e}-4$ & 2.9955 & $(1 / 64,1 / 4)$ & $6.6294 \mathrm{e}-2$ & 3.0369 \\
\hline
\end{tabular}


error estimates in the $L^{2}$ and $H^{1}$ norms for $u$ and $v$ regarding space. The theoretical analysis presented in this paper is different from those in $[3,8,9]$ since we introduce Legendre polynomials and the corresponding Gauss integration rule to study the well-posedness of the STCG scheme and introduce a space-time projection operator to analyze the error estimates in the $L^{2}$ and $H^{1}$ norms for $u-u^{h k}$ and $v-v^{h k}$ such that our theoretical analysis is more concise and understandable. Especially, our techniques can be easily extended to other TRPDEs. Thus, we develop and improve the existing results. Finally, a numerical example is given to validate the feasibility and efficiency of the STCG method. In the future work, we hope that the approach used in this paper will be a foundation for the nonlinear problems, such as the KdV equation, the Klein-Gordon equation, and so on. Moveover, although the STCG method can easily improve the accuracy of approximate solutions, it has lots of degrees of freedom; so in the forthcoming work, we plan to investigate the reduced-order STCG method based on a proper orthogonal decomposition for the wave equation.

Competing interests

The authors declare that they have no competing interests.

Authors' contributions

Both authors contributed equally and significantly in writing this article. All authors read and approved the final manuscript.

\section{Acknowledgements}

This research was supported by National Science Foundation of China grant $(11361035,11271127,11301258)$, Natural Science Foundation of Inner Mongolia (2014BS0101), Postgraduate Scientific Research Innovation Foundation of Inner Mongolia (11200-12110201).

Received: 3 July 2016 Accepted: 21 October 2016 Published online: 10 November 2016

\section{References}

1. French, DA, Schaeffer, JW: Continuous finite element methods which preserve energy properties for nonlinear problems. Appl. Math. Comput. 39, 271-295 (1991)

2. Glassey, RT: Convergence of an energy preserving scheme for the Zakharov equations in one space dimension. Math. Comput. 58, 83-102 (1992)

3. French, DA, Peterson, TE: A continuous space-time finite element method for the wave equation. Math. Comput. 65(214), 491-506 (1996)

4. Aziz, AK, Monk, P: Continuous finite elements in space and time for the heat equation. Math. Comput. 52, 255-274 (1989)

5. Bales, L, Lasiecka, I: Negative norm estimates for fully discrete finite element approximates to the wave equation with nonhomogeneous $L^{2}$ Dirichlet boundary data. Math. Comput. 64, 89-115 (1995)

6. Li, H, Zhao, ZH, Luo, ZD: A space-time continuous finite element method for 2D viscoelastic wave equation. Bound. Value Probl. (2016). doi:10.1186/s13661-016-0563-1

7. Zhao, ZH, Li, H, Luo, ZD: A new space-time continuous Galerkin method with mesh modification for Sobolev equations. J. Math. Anal. Appl. 440, 86-105 (2016)

8. French, DA: A space-time finite element method for the wave equation. Comput. Methods Appl. Mech. Eng. 107, 145-157 (1993)

9. Bales, L, Lasiecka, I: Continuous finite elements in space and time for the nonhomogeneous wave equation. Comput. Math. Appl. 27(3), 91-102 (1994)

10. Adams, RA: Sobolev Spaces. Academic Press, New York (1975)

11. Luo, ZD: The Foundations and Applications of Mixed Finite Element Methods. Chinese Science Press, Beijing (2006) (in Chinese)

12. Brezzi, F, Fortin, M: Mixed and Hybrid Finite Element Methods. Springer, New York (1991)

13. Ciarlet, PG: The Finite Element Method for Elliptic Problems. North-Holland, Amsterdam (1978)

14. Karakashia, O, Makkridakis, C: A time-space finite element method for nonlinear Schrödinger equation: the continuous Galerkin method. Math. Comput. 97(222), 479-499 (1998) 